\title{
Pansofismo y conocimiento en el Prodromus Pansophiae de J.A. Comenius. Una exposición e interpretación de sus presupuestos epistemológicos ${ }^{1}$
}

\section{Pansophismus and Knowledge in J. A. Comenius'Prodromus Pansophiae. Exposition and Interpretation of his Epistemological Ideas}

\author{
Andrés L. JAUME \\ (Universidad de las Islas Baleares)
}

Recibido: $16 / 12 / 2013$

Aceptado: 27/02/2014

\section{Resumen}

El presente artículo expone y analiza las principales ideas epistemológicas abordadas por Comenius en su Prodromus pansophiae. En primer lugar, se subraya el valor e interés filosófico de la obra de Comenius y se relaciona con la problemática intelectual y filosófica de su momento. En segundo lugar, se procede a un análisis del texto destacando su concepción antropológica y la dependencia de una determinada concepción metafísica para el desarrollo de sus ideas epistemológicas. En tercer lugar, se analiza la pansofía como logro metafísico y epistemológico de Comenius. Finalmente, se concluye con un análisis acerca del método presente en el proyecto epistemológico elaborado en el Prodromus.

Palabras clave: Conocimiento, Comenius, Epistemología, Método, Pansofía.

\footnotetext{
1 Este trabajo ha sido posible gracias a una estancia en la Academia Checa de las Ciencias-Instituto de Filosofía durante el mes de agosto de 2012 subvencionada por la Universidad de las Islas Baleares dentro del Programa para el fomento de la investigación. Mis agradecimientos a la colaboración inestimable del Dr. Vladimir Urbánek, director del Departamento de estudios Comeniológicos de la citada institución, y de la Dra. V. Schifferova, por sus consejos y sugerencias sobre el presente texto. Igualmente debo agradecer la colaboración de mis colegas de la Universidad de las Islas Baleares, los Drs. J.L. Llinàs y F. Torres.
} 


\begin{abstract}
This paper examines the main epistemological ideas in Comenius' Prodromus pansophiae. First of all, it is showed the relevance and philosophical interest of Comenius work and its relation with intellectual History of his age. In a second place anthropological and metaphysical background in Comenius' Prodromus is showed. In a third place we analyze pansophism as a Comenius' metaphysical and epistemological achievement. Finally, we conclude with a consideration on the methodical proposal showed in Prodromus.
\end{abstract}

Keywords: Knowledge, Comenius, Epistemology, Method, Pansophia

\title{
Introducción
}

Jan Amos Komenský (1592-1670), latinizado como Comenius, es un autor que, si bien es una de las figuras claves en lo que podríamos denominar la historia del canon de la pedagogía occidental, apenas ocupa un lugar en la Historia de la Filosofía. Las principales traducciones modernas de sus obras se centran en la conocida Didactica Magna (1657), considerada como texto fundacional de la pedagogía moderna, en el Orbis Pictus Sensualium (1658), entre otros escritos dedicados a la enseñanza de las lenguas, y en el conocido Labyrint sveta (El laberinto del mundo y el paraíso del corazón), obra literaria de juventud publicada en $1631^{2}$ y que puede considerarse uno de los textos fundacionales de la literatura checa, si bien en esta la temática filosófica es patente. Sea como fuere, Comenius no es un autor muy conocido en el ámbito filosófico. Si nos atenemos a la idea de un hipotético canon de la filosofía que pudiera venir dado por las Historias de la Filosofía, veremos como brilla por su ausencia. Pero tampoco es un desconocido, sabemos que mantuvo una polémica relación con Descartes, pues ambos hombres sostuvieron un encuentro de apenas pocas horas ${ }^{3}$, que Leibniz le escribió un poema, fruto de su admiración, y que Herder comentó su Pampedia. Sea como fuere, Comenius tiene una obra filosófica que en su momento recibió un cierto reconocimiento y que se presentó, además, como tal. Los escritos pansóficos abordan la temática filosófica propia de la modernidad temprana y, de modo particular, el texto objeto de este estudio, el Prodromus pansophiae, puede leerse como una obra análoga al Discourse de la méthode cartesiano. En efecto, tanto la problemática como las soluciones esbozadas por Comenius mantienen un curioso paralelismo con las ideas que encontramos en la obra de Descartes, si bien desde una perspectiva claramente distinta que tal vez

\footnotetext{
2 Una segunda edición del texto corregida y aumentada fue publicada en Amsterdam en 1663.

3 W. Rood (1970).
} 
pudiera hacernos reflexionar sobre una manera alternativa de encarar el pensamiento moderno, como si Comenius propusiera una modernidad alternativa a la línea que posteriormente se impuso y que, tal vez, quede muy bien perfilada por la figura de Descartes ${ }^{4}$.

La primera edición de lo que hoy conocemos como Prodomus Pansophiae fue publicada en 16375 por Samuel Hartlib en Oxford sin el consentimiento de Comenius bajo el título de Conatuum Comenianorum Praeludia. El texto parece que fue compuesto entre los años 1634-1636 y en vida del autor conoció diversas ediciones. Así, encontramos una edición en 1639, esta vez autorizada por Comenius, en Londres, con un prefacio de Hartlib y con un añadido intitulado por el autor Conatuum Pansophicorum Dilucidatio. Finalmente, el texto volverá a aparecer en 1657 en la edición latina que el autor hiciera de sus principales obras y que publicó en Amsterdam con el título de Opera Didactica Omnia.

El Prodromus fue una obra que causó cierto interés, pues además de tener una buena circulación en su versión latina gracias al propio Hartlib, en 1642 aparecía en Londres una edición en cuarto traducida al inglés y en 1651 otra en octavo, también en Londres y en lengua inglesa. A continuación se expondrán las ideas que acerca del conocimiento podemos encontrar en el Prodromus y que, en cierto modo, representan la primera sistematización del proyecto epistemológico del propio Comenius.

\section{Los escritos pansóficos de Comenius}

Si bien Comenius ha pasado a la historia más como un pedagogo que como un filósofo, hay que decir que su contribución a la historia de la filosofía no es poca. En efecto, dentro de la inmensa colección de escritos de Comenius el volumen de obras de temática exclusivamente filosófica, aun siendo estrictos en separar la pedagogía de la filosofía - algo que en Comenius puede ser discutible-, es muy considerable. Si atendemos al volumen y contenido de las obras, veremos que no estamos solo ante un pedagogo o un filósofo de la educación, sino ante un filósofo con un sistema claro y definido con tesis notables acerca de ontología y epistemología.

Los escritos pansóficos de Comenius están recogidos respectivamente en los vols. 14 y 18 de la edición de sus obras completas editadas por la Academia de las Ciencias de la República $\mathrm{Checa}^{6}$. Entre estos hay que distinguir tres grandes grupos: En primer lugar, los escritos que presentan una primera versión de las ideas pansóficas como son los Praecognita Philosophica -publicados por Turnbull en

\footnotetext{
4 Sobre esta posibilidad interpretativa son interesantes las observaciones de Patočka (2006).

5 El mismo año en que Descartes publica su Discourse de la méthode.

6 J.A. Komenský, Opera Omnia, Praga, Academia, edición iniciada en 1969 que continúa a día de hoy.
} 
19517-, el propio Prodomus en sus diversas ediciones de 1637 y 1639 y los escritos que aparecieron en la década de 1640 como los Pansophica minora Londinensia, el borrador de la Janua rerum reserata de 1643 -que no fue publicado hasta 1681- y los escritos que constituyen la Pansophiae Diatyposis a partir de 1643. De todos estos escritos el que fue publicado, gracias a Hartlib, es el que conocemos como Prodromus pansophiae y que después volvería a aparecer en la edición de algunas de sus obras que el autor hiciera en Amsterdam en 1657 bajo el título de Opera Didactica Omnia. En segundo lugar, tenemos aquellos escritos que aparecen aproximadamente diez años tras la edición de 1657 como son la Prima Philosophia (s.d.), Judicium de Responsione Serarii (1667), Unum necessarium (1668), Janua rerum reserata (1681) o el Triertium catholicum (1681). Finalmente debe considerarse el libro de la Pansophia, obra incluida en su monumental De rerum humanarum emmendatione consultatio catholica que permaneció inédita y no fue publicada hasta mediados del pasado siglo ${ }^{8}$. En esta última obra encontramos quizás la exposición más sistemática y madura de las ideas pansóficas de Comenius. En cualquier caso, y he aquí la principal razón de este estudio, el texto que mayor difusión tuvo y que fue considerado por sus contemporáneos fue el que conocemos como Prodromus pansophiae. En este texto Comenius presenta el proyecto de su pansofía cristiana, proyecto que irá elaborando a lo largo de su vida y el método que permite alcanzarla. Es así un texto de carácter metodológico.

\section{El proyecto del Prodromus pansophiae}

Tanto en el prólogo al lector como en el $\S 98$ del Prodromus, Comenius compara el objetivo de éste con el de sus dos obras precedentes, la Didactica magna y la Janua linguarum. Si aquellas dos obras tenían respectivamente por objeto mostrar el arte de enseñar todo a todos del modo más fácil posible y el vehículo de este aprendizaje -la lengua- del mismo modo, el Prodromus se presenta como una «puerta a las cosas», o un texto de inspiración enciclopedista -pero en modo alguno una enciclopedia- acerca del conocimiento de la realidad. En este sentido, tiene un carácter metodológico compartido con los dos textos ya citados, a la vez que se subraya su especificidad filosófica que hará del mismo un texto de carácter ontológico y epistemológico.

El Prodromus aborda la cuestión del conocimiento en tanto que pansofía. El concepto de pansofía es equivalente al de sabiduría en grado sumo y perfecto. Por pansofía debemos entender el conocimiento perfecto de todo, de Dios mismo incluso. Así las cosas, la pansofía es algo únicamente accesible a Dios y no al hombre,

7 G.H. Turnbull (1947).

8 J.A. Comenius, De rerum humanarum emmendatione consultatio catholica, Praga, Academia, 1966. 
pues solo Dios es, para la tradición y no menos para Comenius, omnisciente. Pero también existe una pansofía humana como señala el propio Comenius en el §108 del Prodromus, a saber, aquello que Dios quiere que el hombre conozca. En el caso de Comenius el problema del conocimiento -o de la sabiduría- se plantea desde una perspectiva múltiple que afecta a dimensiones religiosas, pedagógicas e incluso políticas. De lo que se trata es de reformar los asuntos humanos a través de la educación y el conocimiento. El objetivo es, como señala el título de su gran obra póstuma De rerum humanarum enmendatione, la reforma de los asuntos humanos y su fin último es claramente de orden salvífico como se encarga de recordarnos en diversas obras, entre ellas la Didactica magna ${ }^{9}$. La sabiduría o el conocimiento es algo necesario para el hombre tanto desde un punto de vista práctico como soteriológico. No solo nos permite desenvolvernos en el mundo, sino también alcanzar a $\operatorname{Dios}^{10}$, fin último, según Comenius, de nuestras vidas. Sin embargo, el problema del conocimiento no es menos el problema del método. No se conoce sin más, sino que el conocimiento requiere de un método. En este sentido Comenius, al igual que otros contemporáneos, insiste en la cuestión metodológica como pieza clave para dar una respuesta al problema del conocimiento. El conocimiento, pues, en vías a su obtención, presenta dificultades y para subvenirlas se hace necesario un método y el resultado del mismo es la pansofía. Así, la pansofía no es tanto en este texto una doctrina expuesta como el resultado de la aplicación de un método.

\section{El problema del conocimiento en Comenius}

¿En qué sentido es el conocimiento un problema para Comenius? Nuestro autor considera que el conocimiento es posible y deseable. En este sentido, ni es un escéptico ni parte de presupuestos escépticos. Pero sí subraya la necesidad de reformar tanto el cuerpo del saber como las instituciones que lo dispensan. A lo primero, la reforma del cuerpo del saber, responderá con una propuesta metodológica que podemos muy bien situarla, entre otros textos, en el Prodromus, lo segundo, la reforma de las instituciones que dispensan el saber, será objeto de la Didactica Magna. Reflexionemos, pues, sobre el primer aspecto, la cuestión metodológica que, si bien no es exclusiva del Prodromus, pues también se aborda en la Didactica, sí puede decirse que en ese texto encuentra su lugar natural ${ }^{11}$.

\footnotetext{
9 Cf. Didactica Magna cap.2. «Hominis finem ultimum extra hanc Vitam esse» en Johannes Amos Comenius, Opera Omnia, Vol. 15/1, Praga, Academia. 1986. Las citas referentes a la Didactica Magna se indican mencionando en el capítulo correspondiente a la edición crítica de la Academia de las Ciencias de Praga.

10 Prod. $\$ 7$.

11 Sobre las relaciones entre conocimiento y educación, vid. Jaume (2013).
} 
Comenius pone bastante énfasis en lo que podríamos denominar una antropología filosófica que, además, tiene un papel determinante en la reflexión epistemológica. Tanto es así que no es exagerado afirmar que en Comenius la cuestión epistemológica está subordinada a sus planteamientos antropológicos. Si bien encontramos un desarrollo excelente de las mismos en los primeros capítulos de la Didactica Magna, en el Prodromus pansophiae están no menos presentes. La antropología, por otra parte, explica también por qué el conocimiento es posible y por qué el escepticismo no es una opción razonable. En efecto, el hombre puede conocer porque ha sido creado a imagen y semejanza de Dios y Dios mismo lo ha creado espectador de su sabiduría ${ }^{12}$ que se manifiesta en su creación. Además, el deseo de conocer el mundo es algo connatural al hombre en tanto que criatura. Dios ha puesto en el corazón del hombre el deseo de conocer el mundo «desiderium cordi inditum», de modo que el conocimiento es posible porque Dios quiere que así sea y lo es de modo gradual, no absoluto ${ }^{13}$. De la concepción naturalista del conocimiento antedicha se sigue una determinada doctrina del error, aspecto crucial para determinar una genuina teoría del conocimiento. Entiende el error como falta de perspicacia que, tan pronto como el hombre actúa como criatura racional, es decir, como lo que es, es subsanado. Ahora bien, como se ha señalado, el error también surge del no saber dirigir adecuadamente la razón. De otra manera y siguiendo los presupuestos humanistas de Comenius, el error surge cuando el hombre no cumple con el proyecto de su humanidad. De ahí que la educación sea también una salida al problema del error entendido como ignorancia y sea, a la vez, la causa de esa misma ignorancia. De este modo, los problemas con los que se encuentra el educando o el estudioso en su proceder deben obtener una solución que afecte tanto al cómo enseñar como al cómo aprender. En definitiva, el método requiere de una pedagogía. Para Comenius, pues, no parece necesario distinguir netamente entre Pedagogía y Filosofía. El método es tanto un método de obtener conocimientos como de saberlos exponer y transmitir. Ni la enseñanza se subordina a la investigación ni la investigación puede ser llevada a cabo sin un aprendizaje previo. De este modo, además de aquellas dificultades que conciernen más estrictamente a la naturaleza humana como la fatiga ${ }^{14}$ durante el estudio o la dificultad de memorizar los contenidos a fin de tenerlos presente en la mente o incorporarlos al pensar ${ }^{15}$, encontramos otra serie de dificultades que corresponden al conjunto del conocimiento como puedan ser la prolijidad ${ }^{16}$,

\footnotetext{
12 Cf. Conatuum Comenianorum Praeludia. §45. Las citas concernientes al Prodromus se efectúan mencionando los $\S \S$ que figuran en la obra según la edición crítica preparada por la Academia de las Ciencias de la República Checa: Johannes Amos Comenius, Opera Omnia, Vol. 15/2, Praga, Academia, 1986.

13 Cf. Prod. $\$ 46$.

14 Cf. Prod. $\$ 20$.

15 Cf. Prod. $\S 22$

16 Cf. Prod. $\$ 19$.
} 
vastedad del campo del saber y la falta de experiencia directa con el objeto de estudio ${ }^{17}$. Todas ellas pueden resumirse en la cuestión metodológica. En definitiva, el error radica la ausencia de un método sólido18. Puede decirse así que la cuestión metodológica es la razón de la crisis epistemológica y la solución pasa por reformular el método que es capaz de generar el saber. Es esta una idea claramente ligada al proyecto enciclopédico en el que se inscribe nuestro autor. El Prodromus es un claro ejemplo del proyecto de unir saber enciclopédico bajo principios metodológicos capaces de ampliar substancialmente el conocimiento y no sólo taxonómicos $\mathrm{u}$ $\operatorname{organizativos}^{19}$, pues su proceder apunta tanto a la constitución de la enciclopedia como a la enunciación de los principios de un método que permita ampliar los conocimientos de manera segura y sólida. En suma, la salida al complejo problema epistemológico es la idea de la pansofía entendida como un saber enciclopédico organizado bajo principios generales y universales y este ideal pansófico solo puede ser alcanzado mediante la aplicación de un determinado método.

\section{Definición y alcance del conocimiento}

Como ya se ha señalado, el conocimiento es posible. Consiste este en aprehender la realidad tal cual le es dada al hombre. Comenius tiene una concepción especular del conocimiento ${ }^{20}$, conocer es «ver» las cosas tal como son, así lo afirma en el $\$ 67$ «Cognitio vera tum est, cum res uti sunt, ita cognoscuntur. Si enim aliter Quam sunt cognoscantur, non cognitio erit, sed error», pero el conocer las cosas

17 Cf. Prod. \$23. Comenius apela también a la falta de experiencia directa. De otra manera, uno de los factores de la crisis epistemológica es que no se enseña «per autopsiam» sino que pervive como fuente de justificación epistémica el argumento de autoridad. Uno de los remedios a la crisis epistemológica será, por lo tanto, apelar a los sentidos como fuentes de evidencia. En este punto encontramos una confianza en los sentidos que podemos calificar de optimismo epistemológico, muy lejos de la sospecha cartesiana y más cercana a planteamientos claramente empiristas. En cualquier caso, respecto de otras propuestas epistemológicas modernas, hay que señalar que Comenius es claramente un fundamentalista epistémico, es decir, cree que el conocimiento tiene principios y que las proposiciones se ligan unas a otras a través de relaciones deductivas, como si de una cadena se tratara. De hecho, es la idea de deducción la que nos conduce al último de los puntos, a saber, al problema del método. Comenius considera que el método no es el adecuado ya que no posibilita una aprehensión de la naturaleza de las cosas por la mente humana. Salvo en matemáticas, donde unas proposiciones se siguen de otras con absoluta necesidad y evidencia, el método que siguen las otras ciencias o saberes es confuso. Así, el método que propone incide en la necesidad de ir paso a paso, como si de una cadena deductiva se tratara en la que se va de lo más claro a lo más oscuro de manera gradual y ordenada.

18 Cf. Prod. $\$ 24$

19 Cf. Prod. 26.

20 La idea de la mente como espejo que refleja la realidad tal cual es aparece desarrollada en la Didactica Magna cap. V, 4. 
como son es algo esencial y no solamente fenoménico. Comenius sostiene que algo es conocido cuando se conoce el cómo ha sido hecho, la razón por la que existe. De otro modo, tener conocimiento para Comenius es tener conocimiento de las esencias de las cosas que no es lo mismo que tener conocimiento de las cosas en tanto que contenidos de conciencia o ideas en el sentido moderno del término. Así, Comenius afirmará en el $\S 69$ que todo es hecho conforme a una idea «ad certam quandam rationem» y que estas rationes conforman el ser de las cosas, son «normas rerum» y estas deben ser abstraídas inductivamente 21 con el fin de poder ser consideradas por la mente. El esquema de conocimiento es aprehensivo, el problema es cómo se abstraen las rationes o normae rerum. La respuesta es de corte netamente psicologicista o antropológico, es decir, se apela a la positiva capacidad cognoscitiva humana que justifica en sus reflexiones antropológicas. Al hombre le es dado tener conocimiento del mundo en virtud de sus facultades orgánicas adecuadamente dirigidas por la razón. Lo que hay de nuevo respecto de la concepción aristotélico-escolástica del conocimiento es lo que hay de reformador en Comenius, es decir, el no sujetarse al principio de autoridad y reclamar un papel preeminente a la experiencia directa y personal. Ahora bien, la renuncia al principio de autoridad tiene límites. La autoridad humana puede ser puesta en duda, pero no la divina. En efecto, sin temor de Dios no hay conocimiento posible 22 y la autoridad de la escritura es incuestionable en tanto que es palabra de Dios, principalmente en aquellos asuntos en los que el testimonio de los sentidos o la naturaleza del problema, «in gravioribus materias» dirá, muestran la insuficiencia de la razón.

Ahora bien, una vez admitido que el conocimiento es posible y habiéndolo definido como la captación de lo que es, cabe preguntarse por los objetos del conocimiento. Es decir, se hace necesaria una respuesta a la cuestión acerca de qué puede ser conocido. La respuesta se desprende inmediatamente de su concepción antropológica que claramente refleja sus convicciones religiosas. Si el hombre no solo está en este mundo sino que su fin último está fuera del mismo, es obvio que la presencia de la revelación no puede ser pasada por alto. Por otra parte, del mismo modo que la capacidad sensible del hombre nos remite no menos a su vida terrenal, es patente que esta también constituye un elemento importante cuyo conocimiento no puede ser descuidado. Por lo tanto, para Comenius la realidad entera es objeto de conocimiento 23 . Si Dios, la naturaleza y las creaciones artificiales constituyen la totalidad de lo real son también aquello que puede ser conocido ${ }^{24}$. Dios puede ser conocido en tanto que se revela y se está dispuesto a seguirle. En este sentido, la

\footnotetext{
21 En el Prod. $\$ 80$ leemos: «Atque tales rerum comunes rationes abstrahendae sunt a rebus per inductionem quandam, et ponendae pro normis rerum».

22 Cf. Prod. $\S \S 33-34$.

23 Vid. Schaller (1967), §8.

24 Cf. Prod. § 64.
} 
escritura es fuente de conocimiento. Ahora bien, la naturaleza es algo con lo que el hombre se encuentra y lo artificial es fruto de la creación humana. El acceso cognoscitivo que podamos tener a ambas esferas de la realidad se materializa tan solo cuando este sabe cómo dirigir adecuadamente sus facultades dispuestas naturalmente para este fin pero no menos expuestas al error.

\section{La cuestión del método}

La pansofía es, para Comenius, metafísica y, podría añadirse, una metafísica claramente epistemologizada, pues de lo que se trata es de la sabiduría, en primer lugar, divina y, en segundo, lugar humana. Čapkova (1987) insiste en que la pansofía es metafísica y que Comenius emplea precisamente el adjetivo «pansófico» para distinguir su proyecto de la metafísica tradicional. De hecho no hay que olvidar que el objetivo de Comenius es presentar un sistema metafísico de clara raigambre cristiana y basado en la escritura como fuente de revelación. Pero para alcanzar el conocimiento se hace preciso un método que nos guíe hasta él. El proyecto epistemológico de Comenius se desarrolla, pues, a partir de un método. Comenius en el $\S 82$ del Prodromus insiste en la necesidad de un método que permita construir la pansofía como templo del saber. Ahora bien, ¿en qué consiste la propuesta metodológica de Comenius entendida como organon del conocimiento? En el mismo $\$ 82$ encontramos una solución positiva al problema del conocimiento: un método. Como tal método se compone de un conjunto de reglas que deben ser seguidas y que son formuladas a tenor de las consideraciones acerca de la naturaleza humana y del conocimiento tal como este es posible para el hombre. Comenius así, estipulará en el Prodromus las siguientes reglas:

R.1 (§ 83) Se debe proceder a una anatomía precisa del universo, esto es, a una clasificación exacta o inventario de lo existente.

R.2. (§84) Lo existente debe ser nombrado adecuadamente y de modo exacto, con precisión matemática

R.3. (§85) Deben postularse teoremas y cánones -junto con sus demostraciones pertinentes- a partir de los que deducir o a los que sujetar (subiugare) las divisiones y definiciones de las cosas.

R.4. (§86) Todos estos preceptos deben considerarse claros, unívocos y siempre por sí mismos.

R.5 (§ 87) Los axiomas de la pansofía deben ser reales y prácticos, no demostrados a priori, sino explicitados, pues están impresos en el alma humana.

R.6 (§88) La pansofía no aporta nada nuevo, pues todo lo particular se deduce de los citados principios generales. 
R. 7 (§ 93) Aquello que no pueda ser demostrado apelando a los anteriores principios debe ser reformulado de un modo más claro.

Como puede verse, el método comeniano es una propuesta que guarda un cierto paralelismo con el más conocido método cartesiano. Parte de la consideración de que para conocer es preciso tener noticia de lo que hay (R1) y ser capaz de nombrarlo exactamente (R2). De otro modo, apela a la claridad y distinción a la que refiere Descartes en su primera y segunda reglas, es decir, no dar por válido aquello que no podemos aprehender con evidencia e identificar como básico. En el caso de Comenius, además, lo que podríamos denominar el mandato de la evidencia va ligado al mandato de la exactitud en el nombrar. Las reglas $3^{\mathrm{a}}, 4^{\mathrm{a}}$ y $5^{\mathrm{a}}$ tienen un valor especial. En efecto, podemos ver las referidas reglas como una reformulación de la idea baconiana de «forma», a saber, de aquello que se busca como objetivo de la investigación inductiva que propone Bacon con su lógica. La pansofía no se construye a priori sino que es fruto de la explicitación de aquello que ya está en el alma pero que, a su vez, es lo que está en el mundo, pues el alma es un fiel reflejo del mundo, un microcosmos que reproduce el macrocosmos. Esta formulación de la panarmonía se constituye en principio de inteligibilidad y puede verse como similar al principio racionalista que afirma que el orden de las ideas coincide con el orden de la realidad. De ahí que en la $6^{\mathrm{a}}$ regla añada que no hay nada nuevo sino la explicitación del orden de lo real que es el mismo orden de la mente humana en tanto que microcosmos. De ahí que la necesidad de reforma sea constante, de enderezar aquello que está torcido conforme a un canon o norma de carácter universal y que se manifiesta en el orden natural que el hombre debe seguir. Así, aquello que no queda suficientemente demostrado, como se sugiere en la regla $7^{\mathrm{a}}$, debe ser aclarado o reformulado nuevamente. El método comeniano no es sino el camino que debe seguir la propia razón humana para encontrar una armonía con la razón universal. Se justifica en virtud de su carácter práctico, de que permite conseguir su objetivo con facilidad y sin temor a errar. El método comeniano es un método para bien dirigir los asuntos humanos y alcanzar la dicha de la vida futura. Exhibe un orden, el de la propia realidad que se señala como objeto de estudio.

Pero, ¿en qué se fundamenta en última instancia el método? Es aquí donde claramente entran en escena las ideas metafísicas de Comenius. En cierto modo la respuesta ya ha sido dada. Queda claro que solo si el hombre sabe cómo conducir su propia razón puede alcanzar el conocimiento. Y aplicar la razón correctamente no es otra cosa que aplicar el método. Pero, ¿por qué el método debería conducirnos al conocimiento? Para Comenius la respuesta es muy similar a la que posteriormente ofrecerán los racionalistas, en última instancia encuentra su fundamento en una antropología que sitúa al hombre como microcosmos de la realidad y que no deja de lado su condición de criatura hecha a imagen y semejanza de Dios. Así, el méto- 
do es la propia encarnación del orden o armonía universal. El orden epistemológico es dependiente del orden ontológico 25 . Dicho de otra manera, el método no es un artificio, sino el buen proceder de la razón conforme a la armonía universal que es el fundamento de todo lo existente ${ }^{26}$. La posibilidad misma del conocimiento se basa en una metafísica creacionista de corte platónico agustiniano ${ }^{27}$. En efecto, lo existente responde a un plan o arquetipo que en última instancia es divino. Las cosas artificiales imitan a las naturales, que a su vez imitan a las ideas en la mente de Dios, que Dios crea a partir de sí mismo28. Comenius llegará a afirmar en el $§ 73$ que «Et quia de ideis Divinae mentis Omnia participant, fit ut inter se quoque participent et sibi invincem proportionata sint»; la raigambre platónico-agustiniana no puede ser más clara. La cognoscibilidad es posible en la medida en que se da una comunicación entre las diversas criaturas en tanto que criaturas que comparten un mismo origen y responden al mismo modelo, las ideas divinas.

\section{Conclusiones}

Si bien Comenius ha pasado a la historia antes como un pedagogo que como un filósofo, una lectura atenta de sus escritos pansóficos demuestran su lugar indudable dentro del ámbito estrictamente filosófico. Las ideas pansóficas tuvieron una relativa buena acogida al principio y fueron progresivamente abandonadas posteriormente. Podemos aducir como una de las causas el auge progresivo de la nueva ciencia y de lo que podríamos denominar el triunfo de cierta interpretación de la modernidad -la cartesiana-. En efecto, el principal problema de la pansofía, como se ha señalado en las páginas anteriores, era la integración de ciencia y fe, algo que en un ámbito fuertemente católico no podía triunfar en modo alguno debido a la preeminencia y poder de la Iglesia Católica Romana. La estrategia que seguiría Descartes -como ejemplo claro de lo que después sería la interpretación de la Modernidad dominante- insistía en la separación de ambos ámbitos.

Entre los aspectos más sobresalientes y definitorios de Comenius en el Prodromus cabe destacar los siguientes: En primer lugar, su concepción reformista tanto del conocimiento en un sentido general como de la institución educativa en tanto que dispensadora del mismo y formadora del sujeto cognoscente. El conocimiento, para Comenius, es un bien preciado y deseable con un valor práctico y sote-

\footnotetext{
25 Vid. K. Schaller (1967). En este punto Schaller es preclaro.

26 Prod. §75: «Fundamentum ergo rerum omnium ut condendarum, sic cognoscendarum harmonia est».

27 Comenius cita a Agustín en numerosas ocasiones, tal como ha mostrado V. Balík (2007). Que Comenius leyó y estudió a Agustín directamente es algo que queda fuera de toda duda. Otros autores que han abordado la cuestión son Čapkova (2007), K. Floss (2007), M. Steiner (2007) et alii en el número monográfico dedicado a este tópico de Studia Comeniana et historica XXXVII/2007.

28 Cf. Prod. $\S \S 69-73$.
} 
riológico indiscutible, como se ha puesto de manifiesto en las páginas precedentes. En segundo lugar, la multifactorialidad del conocimiento que hace que Comenius plantee sus intentos reformistas no solo a un nivel personal o individual de cada sujeto, sino colectivo y, de manera más concreta, a través de las instituciones educativas. De ahí la vertiente claramente pedagógica de nuestro autor. En tercer lugar, cabe destacar el problema metodológico. Este es uno de los grandes problema de la época y es, además, objeto de una parte destacada del texto que nos ha ocupado. En efecto, Comenius está en consonancia con los intentos reformistas y de innovación metodológica de Bacon y Descartes. Finalmente, y como rasgo claramente distintivo de la filosofía comeniana, debe enfatizarse la tradición enciclopedista en la que se inserta la obra de Comenius y que, si bien en el siglo XVII no triunfará, sí resurgirá en el XVIII. En efecto, Comenius entiende el conocimiento también como enciclopedia -que no como amasijo desorganizado de saberes-. En conclusión, puede decirse que el Prodromus pansophiae es así una salida a la crisis epistemológica propia de la primera modernidad.

\section{Referencias bibliográficas}

\section{Fuentes}

Comenius, J.A., De rerum humanarum emmendatione consultatio catholica, Praga, Academia, 1966.

Comenius, J.A., Opera Omnia, Vol. 14, Praga, Academia, 1974.

Comenius, J.A., Opera Omnia, Vol. 15/1, Praga, Academia, 1986.

Comenius, J.A., Opera Omnia, Vol. 15/2, Praga, Academia, 1986.

Comenius, J.A., Opera Omnia, Vol. 18, Praga, Academia, 1974.

\section{Estudios}

BALík, V. :«Citáty raně církevních Otců v díle J.A. Komenského/ Quotattions from the Early Church Fathers in the Works of Johann Amos Comenius», Studia Comeniana et Historica XXXVII, 2007, pp. 15-31.

Burke, P.: Historia social del conocimiento, Paidós, Barcelona, 2002.

ČAPKOVÁ, D.: «The reception given to the Prodromus pansophiae and the methodology of Comenius», Acta Comeniana 7 (XXXI), 1987, pp. 37-59.

ČAPKOVÁ, D.: «The Philosophical significance of the work of Comenius», Acta Comeniana 8(XXXII), 1989, pp. 5-16.

ČERVENKA, J.: «Die Grundlagen der pansophischen Idee des Johann Amos Comenius», Acta Comeniana (XXV), 1969, pp. 77-85. 
Floss, P.: «Komenskýs Erkenntnislehre und einblick in seine Metaphysik» Colloquia Comeniana vol.II, 1969, pp. 83-91.

JAUME, A. L.: «Conocimiento, método y formación en Comenius y Descartes», en Cuadernos Salmantinos de Filosofia, vol. 40, 2013, pp. 85-99.

Kunna, U.: Das "Krebsgeschwür der Philosophie» Komenskýs Auseinandersetzung mit dem Cartesianismus, Academia Verlag. Sankt Augustin, 1991.

Mout, N.: «Comenius, Descartes and Dutch Cartesianism», Acta Comeniana 3(XXVII), 1972, pp. 239-243.

PAтос̌KA, J.: Andere Wege in die Moderne. Studien zur europäischen Ideengeschichte von der Reinassance bis Romantik, edición a cargo de L. Hagedorn, Köningshausen \& Neuman, 2006.

Popkin, R. H.: The History of Scepticism from Erasmus to Spinoza, University of California Press, Los Angeles, 1979.

Rood, W.: Comenius and the Low Countries, Van Gendt \& Co. Amsterdam, 1970.

Rossi, P.: Clavis Universalis. Arti della memoria e logica combinatoria da Lullo a Leibniz, Il Mulino, Bolonia, 2002.

Schaller, K.: Die Pädagogok des Johann Amos Comenius um die Anfänge des pádagogischen Realismus im 17. Jahrhundert, Quelle \& Meyer, Heidelberg, 1967.

Schaller, K.: Comenius 1992. Gesammelte Beiträge zum Jubiläumsjahr, Academia Verlag, Sankt Augustin, 1992.

Turnbull, G.H.: Hartlib, Dury and Comenius. Gleanings from Hartlib's Papers, University Press of Liverpool, Londres, 1947.

UrbáneK, V.: «The Network of Comenius Correspondents», Acta Comeniana 12(XXXVI), 1997, pp. 63-78.

VAN DE VEN, J. y Bos, E.J.: «Se nihil daturum- Descartes's unpublished judgement of Comenius's Pansophiae Prodromus (1639) », British Journal for the History of Philosophy 12 (3), 2001, pp. 369-386.

Andrés L. Jaume

Universidad de las Islas Baleares

andres.jaume@uib.es 